\title{
Analysis of Funding Source and Spin in the Reporting of Studies of Intravitreal Corticosteroid Therapy for Diabetic Macular Edema: A Systematic Review
}

This article was published in the following Dove Press journal: Clinical Ophthalmology

\author{
Harrish Nithianandan (ID) \\ Ajay E Kuriyan ${ }^{2}$ \\ Michael J Venincasa $\mathbb{D}^{3}$ \\ Jayanth Sridhar ${ }^{3}$
}

'Department of Ophthalmology \& Visual Sciences, McGill University, Montreal, Quebec, Canada; ${ }^{2}$ Retina Service, Wills Eye Hospital, Philadelphia, PA, USA;

${ }^{3}$ Department of Ophthalmology, Bascom Palmer Eye Institute, Miller School of Medicine, University of Miami, Miami, FL, USA
Correspondence: Jayanth Sridhar Email jsridharl@med.miami.edu

\begin{abstract}
Purpose: This systematic review examined the relationship between industry funding and the presence of spin in high-impact studies evaluating intravitreal corticosteroid therapy for diabetic macular edema.

Methods: This systematic review was conducted in accordance with the Preferred Reporting Items for Systematic Reviews and Meta-Analyses (PRISMA) statement. MEDLINE and Embase were systematically searched from inception through July 16, 2018, for randomized controlled trials and meta-analyses investigating the treatment of patients with diabetic macular edema using intravitreal corticosteroid therapy. Only studies published in English journals with an impact factor greater than 2 as per the Clarivate Analytics 2017 Journal Citation Report were included. The authors independently assessed study quality, funding source and the presence of reporting bias using a standardized datasheet.
\end{abstract}

Results: Title and abstract screening were completed on 7158 unique hits and full-text review yielded 44 included studies. Overall, there was correspondence between the wording of abstract conclusions and study results in $41 / 44$ (93\%) articles. Correspondence between abstract conclusions and significance of main outcome was present in 14/14 (100\%) industryfunded and 27/30 (90\%) nonindustry-funded studies. The odds ratio of industry funding being associated with noncorrespondence was 0.27 (95\% CI: 0.01-5.61, $\mathrm{p}=0.54)$. The most common reason for noncorrespondence was the failure to mention rates of steroid-related intraocular pressure elevation.

Conclusion: The results of this systematic review indicate that biased abstract outcome reporting is rare in published randomized controlled trials and meta-analyses of intravitreal corticosteroid therapy for diabetic macular edema. Biased reporting was not associated with the presence of industry funding or a conflict of interest.

Keywords: corticosteroids, diabetic retinopathy, intravitreal therapy, macular edema, systematic review

\section{Introduction}

Clinical research trials sponsored by the pharmaceutical industry have profound impacts on the practice of medicine. ${ }^{1}$ The industry can support a number of trials at all stages of a product's life with significant financial investment assigned to the innovation. $^{2}$ Therefore, there is potential for considerable monetary loss if the results and conclusions of these trials are unfavourable for the sponsor. ${ }^{1,3}$ A number of systematic reviews have documented industry sponsorship of drug 
studies to be associated with findings favourable to the sponsor. $^{4-8}$ More recently, a review written by the Cochrane Collaboration found there to be less correspondence between the results and conclusions of industryfunded studies when compared to non-industry-funded studies. $^{1}$

In an era of rapidly developing therapies, physicians often rely on the peer-reviewed literature - especially the abstracts of published studies - to remain well informed about their respective fields of practice. It is therefore of paramount importance to evaluate outcome reporting bias in study abstracts. This was assessed in the ophthalmic literature by Alasbali and colleagues, who found industryfunded studies on the ocular hypotensive efficacy of topical prostaglandin analogues to be more likely to report proindustry abstract conclusions, which often did not correspond with studies' results. ${ }^{9}$ Our group performed a similar analysis of randomized clinical trials on the efficacy of intravitreal anti-vascular endothelial growth factor (anti-VEGF) therapy for retinal vein occlusion published in high-impact journals and did not find industrysponsorship to be associated with an increased rate of reporting bias. ${ }^{10}$ The focus on high-impact journals allowed the authors to capture journals that were most likely to be referred to by physicians, and the results of this study were reassuring given the rapid adoption of antiVEGF therapy for a number of ocular conditions.

Treatment of diabetic macular edema (DME) remains controversial among vitreoretinal specialists. ${ }^{11,12}$ Given the increased recognition of the role of inflammation in the development of DME, intravitreal corticosteroid therapy has been shown to provide promising anatomical and visual benefits, especially when compared to laser therapy. ${ }^{13-15}$ Compared to anti-VEGF therapy, intraocular corticosteroids do carry class-specific risks such as cataract progression and ocular hypertension that theoretically could be downplayed in abstract presentation. ${ }^{15}$ The purpose of this study was to examine the relationship between industry funding and the presence of spin in high-impact studies assessing the efficacy and safety of intravitreal corticosteroid therapy for DME.

\section{Methods}

This systematic review was conducted in accordance with the Preferred Reporting Items for Systematic Reviews and Meta-Analyses (PRISMA) statement. ${ }^{16}$ Approval from an Institutional Review Board was not required for this study as no human subjects were involved, and analysis was based upon information from published literature.

\section{Search Methods}

Ovid Medline and Ovid Embase were searched from inception through July 16, 2018, for studies investigating the treatment of patients with diabetic macular edema with intravitreal corticosteroid therapy (ie, triamcinolone, fluocinolone, or dexamethasone) used in at least one of their treatment arms. The search strategy (Appendix 1) was designed to generate studies published in English journals with an IF greater than 2 as per the Clarivate Analytics 2017 Journal Citation Report. ${ }^{17}$ The inclusion of studies published in journals with IF greater than 2 is presumed to reflect studies more likely to be read by clinicians when browsing recent medical literature.

\section{Study Selection}

Search results were imported into DistillerSR (Evidence Partners; Ottawa, Canada) to manage all identified records. Title and abstract screening was completed to select for studies that were primary RCTs and meta-analyses. Fulltext screening was then completed to select for studies that reported on main outcomes of visual acuity, retinal thickness, and/or complications. Studies that published secondary or sub-analyses on previously published RCTs were excluded. Figure 1 outlines the flow of study selection as per the PRISMA statement. ${ }^{16}$

\section{Data Collection and Analysis}

Our methods of study evaluation and data extraction have been previously described. ${ }^{10}$ Study quality was assessed with the scoring scale utilized by Alasbali et al (2009) and is outlined in Table 1. ${ }^{9}$ Correspondence between significance of the main outcome measure result and abstract conclusion was assessed by surveying whether the wording of the abstract conclusion matched the statistical analysis of the results as they pertained to the main outcome measure(s). Following independent data extraction, all discrepancies on the standardized data sheet were resolved by unanimous agreement amongst the authors (HN, AK, JS). One author (HN) also collected objective data on the included studies: sample size, source of funding (industry vs non-industry), whether the publication was authored by an industry employee as reported in the manuscript, and whether any of the co-authors had a potential conflict of interest (COI). The presence of a potential author COI was determined by assessing the study's disclosure statement and was defined as any 


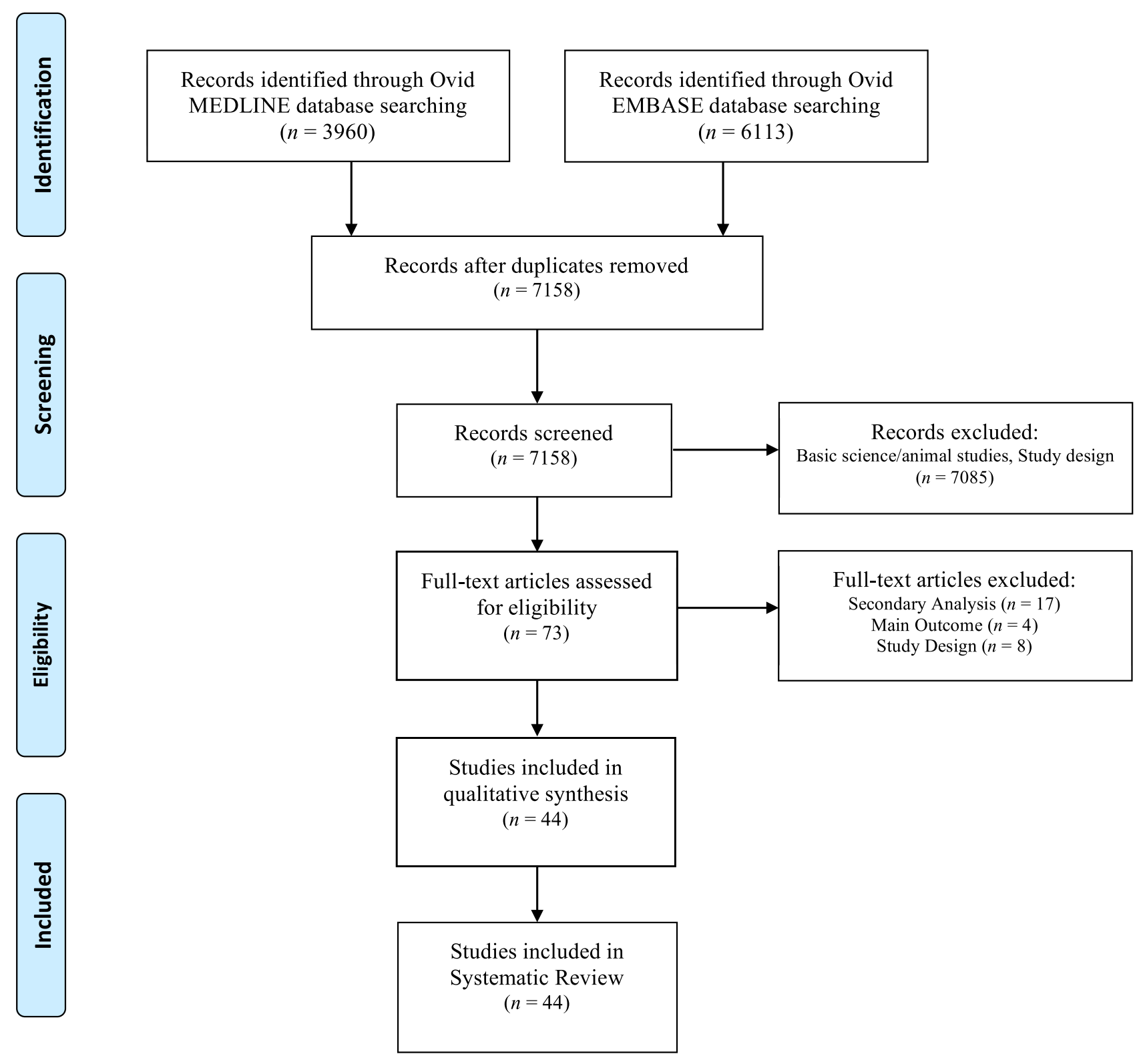

Figure I Selection of randomized clinical trials and meta-analyses.

previous relationship between a co-author and the company that manufactured the drug intervention(s) being studied. Corresponding authors of the included studies were contacted if any of the objective information was not evident as part of the published article.

The primary outcome of the present study was the association between funding source and the correspondence between the studies' abstract conclusion and statistical significance of their main outcome, expressed as an odds ratio. Exploratory secondary analyses were also performed to determine any associations between our variables of interest, including study sample size, journal impact factor and the presence of any COI. Statistical analysis included the Fisher exact test for categorical data, and the Mann-Whitney $U$-test/ Kruskal-Wallis test or Student's $t$-test/one-way ANOVA for numerical data, as appropriate. A p-value $<0.05$ was considered statistically significant. All data were extracted and stored in Microsoft Excel software (Microsoft; Redmond, WA). Statistical analysis was performed using SPSS (IBM Corp; Armonk, New York; software version 22).

\section{Results}

The original search of both databases yielded 10,073 articles, reduced to 7158 following the removal of duplicates. After title and abstract screening, the full texts of 73 articles were completed. Twenty-nine articles were then excluded due to 
Table I Criteria Utilized for Grading of Study Quality ${ }^{9}$

\begin{tabular}{|c|c|}
\hline Quality Score & Criteria \\
\hline $\begin{array}{l}\text { I: Meta-analysis (To assign this } \\
\text { level, all of the following criteria } \\
\text { must be met). }\end{array}$ & $\begin{array}{l}\text { I. The paper reports } \\
\text { a comprehensive search for } \\
\text { evidence. } \\
\text { 2. The authors avoid bias in } \\
\text { selecting articles for inclusion. } \\
\text { 3. The authors assess each article } \\
\text { for validity. } \\
\text { 4. The paper reports clear } \\
\text { conclusions that are supported } \\
\text { by the data and appropriate } \\
\text { analysis. }\end{array}$ \\
\hline $\begin{array}{l}\text { I: Large RCT (To assign this } \\
\text { level, all of the following criteria } \\
\text { must be met). }\end{array}$ & $\begin{array}{l}\text { I. Patients were randomly } \\
\text { allocated to treatment groups. } \\
\text { 2. Follow-up was at least } 80 \% \\
\text { complete. } \\
\text { 3. Both the patients and the } \\
\text { investigators were blind to the } \\
\text { treatment the patient received. } \\
\text { 4. Patients were analyzed in the } \\
\text { treatment groups to which they } \\
\text { were assigned. } \\
\text { 5. The sample size was large } \\
\text { enough to detect the outcome of } \\
\text { interest. }\end{array}$ \\
\hline 2: RCT & $\begin{array}{l}\text { RCT or overview that did not } \\
\text { meet level I }\end{array}$ \\
\hline
\end{tabular}

Abbreviation: RCT, randomized controlled trial.

being a secondary analysis $(n=17)$, analysis of a main outcome not relevant to the present study $(n=4)$, or inadequate study design $(n=8)$. Therefore, 44 (41 RCTs and 3 metaanalyses) publications were included in the present analysis (Figure 1). ${ }^{18-61}$ Of these 44 studies, 36 (82\%) were of higher impact factor ( $I F \geq 3)$, and $31(70 \%)$ were assigned a study quality score of 2 . Fourteen (32\%) received industry funding, five $(11 \%)$ had an author who was an industry employee, and $17(39 \%)$ had an author(s) with a potential COI. Table 2 outlines characteristics of the included studies.

\section{Correspondence Between Main Outcome Measure and Abstract Conclusion}

Statistically significant main outcome measures were present in 26 of $44(59 \%)$ of the included studies. There was correspondence between wording of abstract conclusions and study results in 41 of $44(93 \%)$ articles. Reasons for non-correspondence included the failure to mention high rates of steroid-related intraocular pressure (IOP) elevation $(n=2)^{41,42}$ and the implication of safety despite a case of endophthalmitis in a small sample $(n=1)$ (Table 2$).{ }^{52}$

\section{Funding}

Among the 14 studies that received industry funding, two were funded by Alimera Sciences, Inc., ${ }^{27,30}$ eight studies by Allergan, Inc., ${ }^{18-22,24,54,55}$ three by both Allergan, Inc. and Genentech, Inc., ${ }^{23,26,48}$ and one study was funded by Bausch \& Lomb, Inc. ${ }^{28}$ Eighteen studies received funding from a nonindustry sponsor.

\section{Comparing Industry-Funded versus Nonindustry-Funded Studies}

A statistically significant main outcome measure was reported in 8 of $14(57 \%)$ industry-funded studies and in 18 of $30(60 \%)$ nonindustry-funded studies ( $\mathrm{p}=1.00$, Fisher exact test). Correspondence between abstract conclusions and significance of main outcome was present in 14 of $14(100 \%)$ industry-funded and 27 of 30 (90\%) nonindustry-funded studies. The odds ratio of industry funding being associated with noncorrespondence was 0.27 (95\% CI: 0.01 to $5.61, \mathrm{p}=0.54$ ). Industry-funded studies had significantly greater sample sizes $(\mathrm{p}=0.01)$, but similar mean study quality $(\mathrm{p}=0.50)$ and journal impact factor $(\mathrm{p}=0.14)$ when compared to the nonindustryfunded studies. These data are summarized in Table 3.

\section{Comparing Higher-Impact versus Lower-Impact Publications}

When publications were stratified by journal IF into a "highimpact" group $(\mathrm{n}=36)$ with IF $\geq 3$ and a "low-impact" group ( $n=8)$ with $\mathrm{IF}<3$, statistically significant main outcome measures were reported in 21 of 36 (58\%) highimpact publications and in 5 of $8(63 \%)$ of low-impact publications $(\mathrm{p}=1.00)$. Correspondence between abstract conclusions and significance of the main outcome was present in 33 of $36(92 \%)$ high-impact publications and in 8 of 8 $(100 \%)$ of low-impact publications $(\mathrm{p}=1.00)$. When comparing high-impact and low-impact publications there were no significant differences in rates of significant main outcome measures $(p=1.00)$, rates of industry funding $(p=1.00)$, rates of author COI $(p=0.76)$, sample size $(p=0.87)$, or study quality $(\mathrm{p}=0.68)$. These data are summarized in Table 4 .

\section{Comparing COI-Present versus COl-Absent Publications}

After compiling responses from corresponding authors with published disclosures, 17 studies had a COI, 22 had no COI 


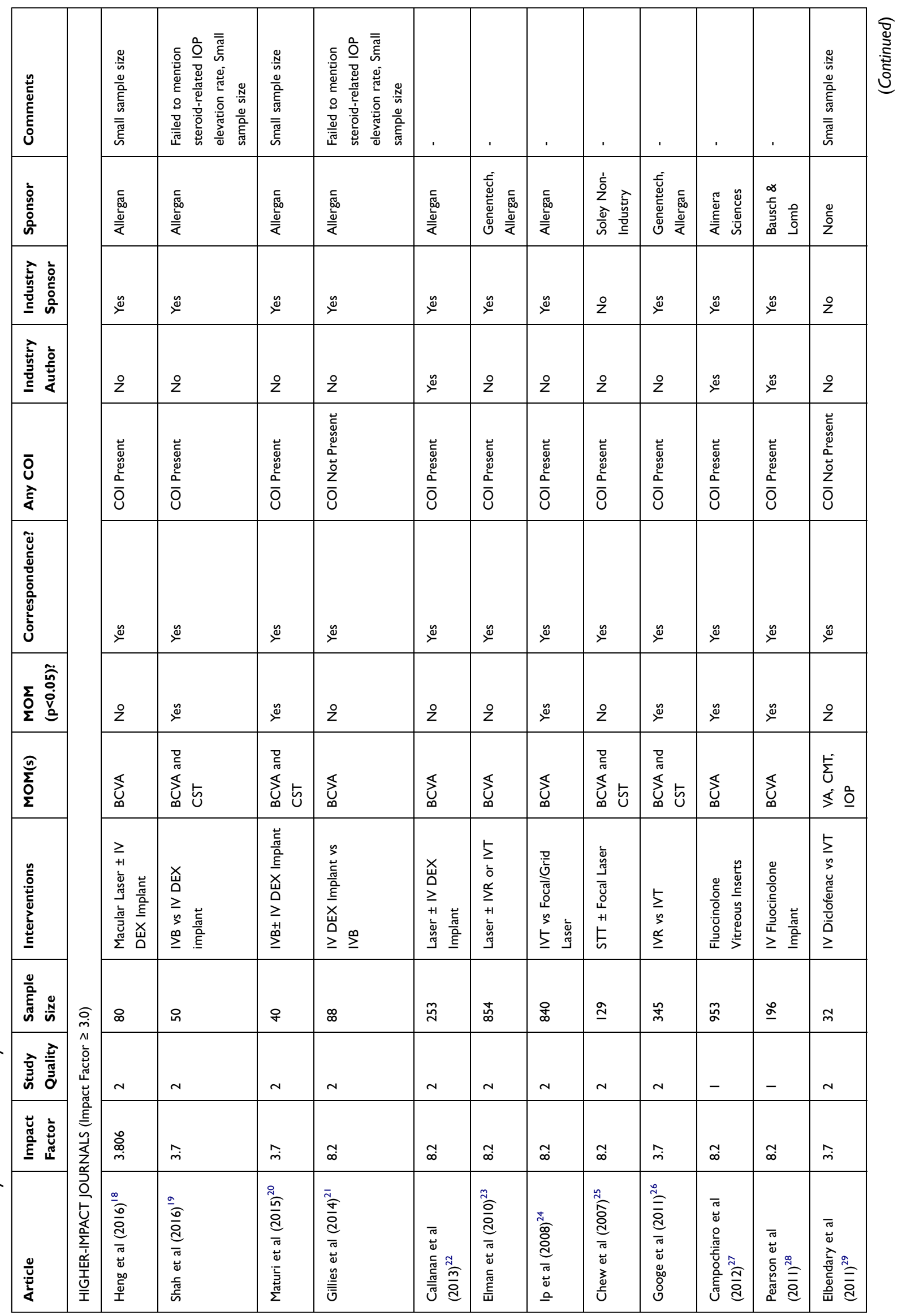




\begin{tabular}{|c|c|c|c|c|c|c|c|c|c|c|c|}
\hline 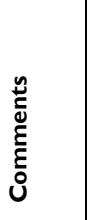 & . & 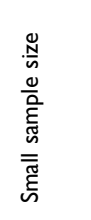 & 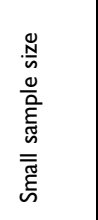 & 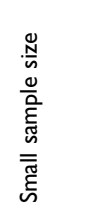 & 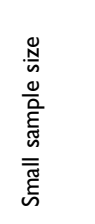 & & 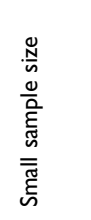 & 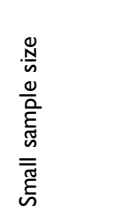 & 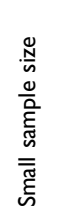 & 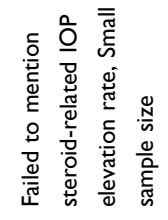 & . \\
\hline $\begin{array}{l}\vdots \\
\bar{\omega} \\
\vdots \\
\vdots \\
\text { nे }\end{array}$ & 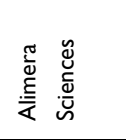 & 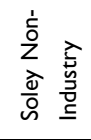 & 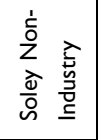 & $\begin{array}{l}0 \\
\stackrel{0}{0} \\
z\end{array}$ & 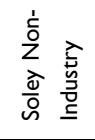 & 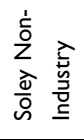 & 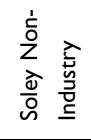 & $\begin{array}{l}\stackrel{0}{\circ} \\
\stackrel{0}{z}\end{array}$ & 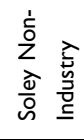 & 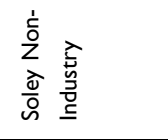 & 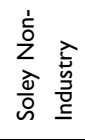 \\
\hline 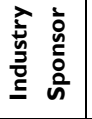 & 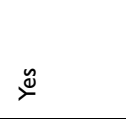 & $\stackrel{\circ}{z}$ & $\stackrel{\circ}{z}$ & $\stackrel{\circ}{z}$ & $\stackrel{0}{z}$ & $\stackrel{\circ}{z}$ & ż & ¿o & $\stackrel{0}{z}$ & \& & $\stackrel{\circ}{z}$ \\
\hline 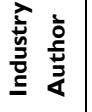 & $\stackrel{\mathscr{u}}{\check{L}}$ & $\stackrel{\circ}{z}$ & $\stackrel{\circ}{z}$ & $\stackrel{\circ}{z}$ & $\stackrel{\circ}{z}$ & $\stackrel{\circ}{z}$ & $\stackrel{\circ}{z}$ & $\stackrel{0}{z}$ & $\stackrel{0}{z}$ & $\stackrel{\circ}{z}$ & $\stackrel{\circ}{z}$ \\
\hline $\begin{array}{l}\overline{0} \\
\text { ত̀ }\end{array}$ & 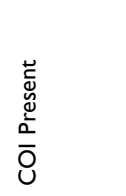 & 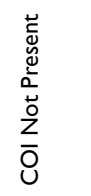 & 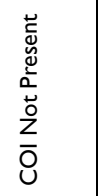 & 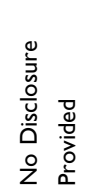 & 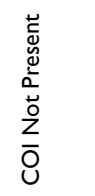 & 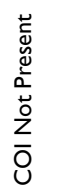 & 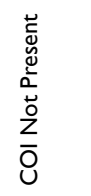 & 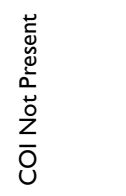 & 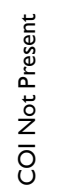 & 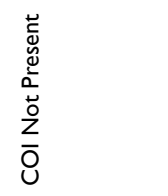 & 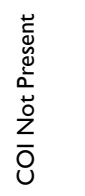 \\
\hline 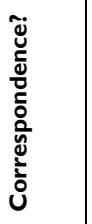 & $\stackrel{\mathscr{\Perp}}{\check{\nu}}$ & $\stackrel{\mathscr{\Perp}}{\check{\nu}}$ & $\stackrel{\check{\Perp}}{\check{\nu}}$ & 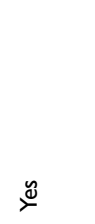 & $\stackrel{\check{\Perp}}{\check{\nu}}$ & $\stackrel{\check{\Perp}}{\check{\nu}}$ & $\stackrel{\check{\Xi}}{\check{\nu}}$ & $\stackrel{\check{\varpi}}{\check{\nu}}$ & $\stackrel{\mathscr{y}}{=}$ & $\stackrel{\check{\Perp}}{\check{\nu}}$ & $\stackrel{\check{\varpi}}{\check{\nu}}$ \\
\hline 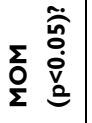 & $\stackrel{\mathscr{u}}{\check{L}}$ & $\stackrel{\mathscr{x}}{\varkappa}^{\mathscr{y}}$ & $\stackrel{\mathscr{x}}{\succ}$ & $\stackrel{\circ}{z}$ & 으 & $\stackrel{\stackrel{y}{\tau}}{=}$ & 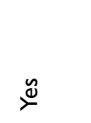 & 우 & 우 & $\stackrel{\mathscr{y}}{\tau}$ & 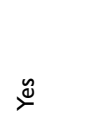 \\
\hline$\frac{\sqrt[n]{n}}{\sum_{0}}$ & 勇 & 勇 & $\sum_{\substack{5 \\
\sum}}^{\frac{0}{0}}$ & $\sum_{\substack{S\\
}}^{+5}$ & 勇 & 勇 & 离 & 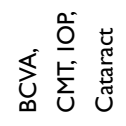 & 胥 & 总 & 勇 \\
\hline 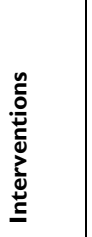 & 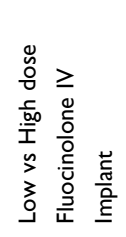 & 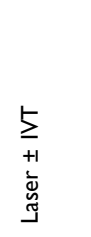 & 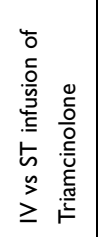 & 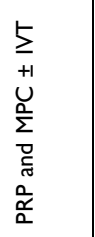 & 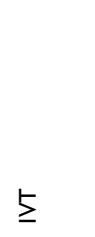 & 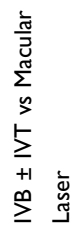 & 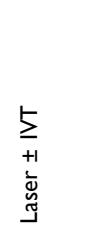 & 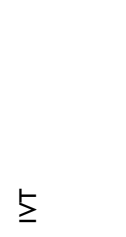 & 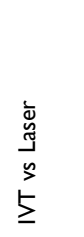 & $\begin{array}{l}\sum_{2}^{\infty} \\
\stackrel{n}{5} \\
\geq\end{array}$ & 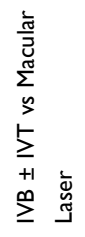 \\
\hline 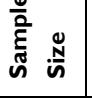 & ณू & $\stackrel{+}{\infty}$ & $\stackrel{ \pm}{\sim}$ & $\stackrel{\circ}{m}$ & $\bar{\infty}$ & 요 & F & $\mathcal{F}$ & $\infty_{\infty}^{\infty}$ & $\stackrel{\sim}{\sim}$ & $\underline{\underline{o}}$ \\
\hline 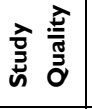 & - & - & $N$ & N & - & - & N & $N$ & $N$ & $N$ & - \\
\hline 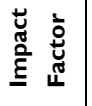 & $\infty$ & $\infty$ & $\hat{m}$ & $\hat{m}$ & $\stackrel{\infty}{m}$ & $\stackrel{\infty}{\infty}$ & 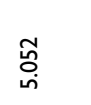 & $\hat{m}$ & 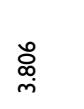 & 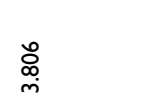 & $\hat{m}$ \\
\hline 造 & 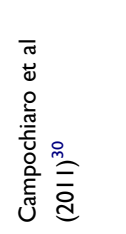 & 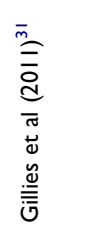 & 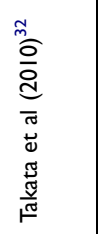 & 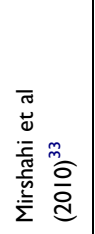 & 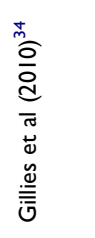 & 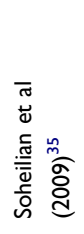 & 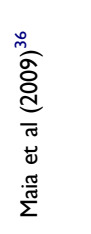 & 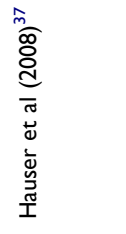 & 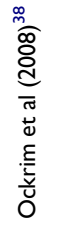 & 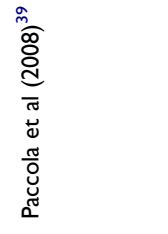 & 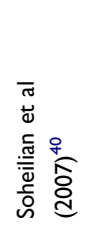 \\
\hline
\end{tabular}




\begin{tabular}{|c|c|c|c|c|c|c|c|c|c|c|}
\hline 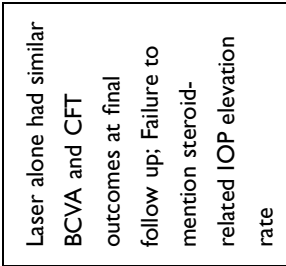 & 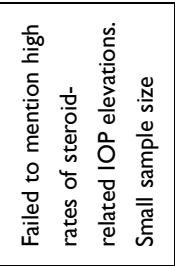 & 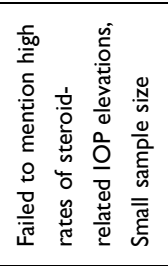 & 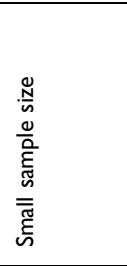 & 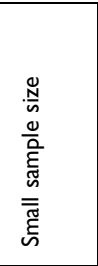 & 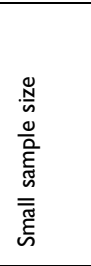 & 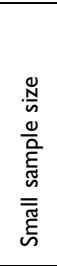 & & 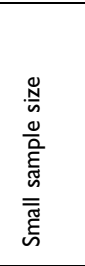 & 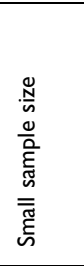 & 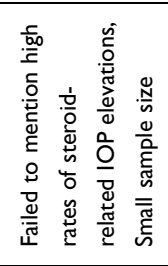 \\
\hline 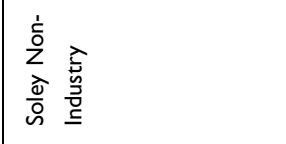 & 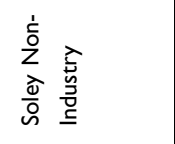 & $\begin{array}{l}\stackrel{0}{\circ} \\
\stackrel{0}{z}\end{array}$ & 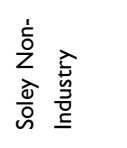 & $\begin{array}{l}\stackrel{0}{0} \\
\text { Zे }\end{array}$ & 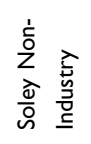 & $\begin{array}{l}0 \\
\stackrel{0}{0} \\
z\end{array}$ & 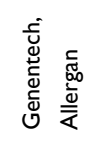 & $\begin{array}{l}\stackrel{0}{0} \\
\text { Zे }\end{array}$ & $\begin{array}{l}\stackrel{0}{\tilde{0}} \\
\text { ż }\end{array}$ & 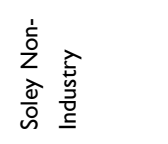 \\
\hline ㅇ & zo & zo & 우 & zo & 우 & zo & $\stackrel{\mathscr{y}}{\nu}$ & zo & $\stackrel{\circ}{z}$ & z̊ \\
\hline$\stackrel{\circ}{z}$ & $\stackrel{0}{z}$ & $\stackrel{0}{z}$ & $\stackrel{0}{z}$ & zo & zo & zo & $\stackrel{\circ}{z}$ & $\stackrel{\circ}{z}$ & zo & zo \\
\hline 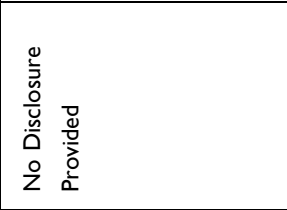 & 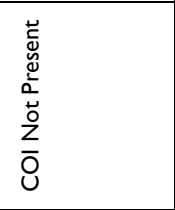 & 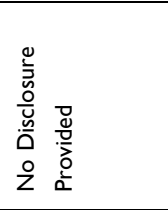 & 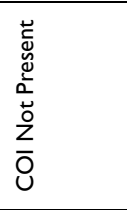 & 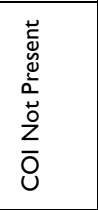 & 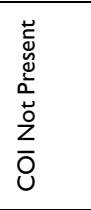 & 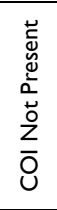 & 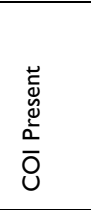 & 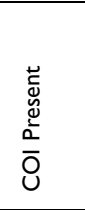 & 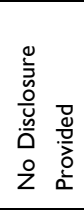 & 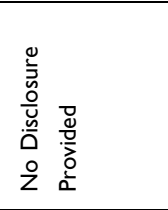 \\
\hline$\stackrel{0}{z}$ & 으 & 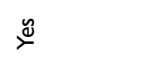 & 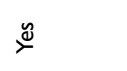 & $\stackrel{\check{\nu}}{\check{\nu}}$ & $\stackrel{\check{\varpi}}{\check{\nu}}$ & $\stackrel{\check{\nu}}{\sim}$ & $\stackrel{\check{\Xi}}{\check{\nu}}$ & $\stackrel{\check{\nu}}{\check{\nu}}$ & $\stackrel{\check{\Xi}}{\check{\nu}}$ & $\check{\mathscr{\nu}}^{\ddot{\theta}}$ \\
\hline$\stackrel{\circ}{z}$ & $\stackrel{\mathscr{u}}{\tau}$ & $\stackrel{\circ}{z}$ & $\check{\nu}^{\underline{y}}$ & $\stackrel{\tilde{y}}{\tau}$ & $\stackrel{y}{\tau}$ & $\stackrel{\tilde{x}}{\tau}$ & $\stackrel{\circ}{z}$ & $\stackrel{\mathscr{E}}{\tau}$ & $\stackrel{\mathscr{y}}{\tau}$ & 우 \\
\hline 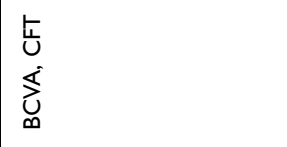 & 造 & $\sum_{U}^{E}$ & 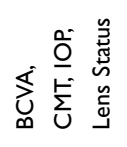 & $\frac{0}{\underline{0}}$ & $\sum_{D}^{S}$ & 勇 & 志 & 恿 & 㫐 & 勇 \\
\hline 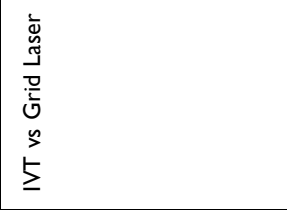 & $\underline{5}$ & $\underline{5}$ & $\begin{array}{l}5 \\
5 \\
5 \\
5\end{array}$ & $\underline{5}$ & $\begin{array}{l}5 \\
5 \\
5 \\
5 \\
5\end{array}$ & 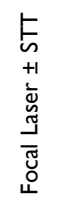 & 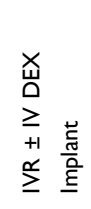 & 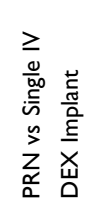 & $\begin{array}{l}\infty \\
\geq \\
2 \\
2 \\
2\end{array}$ & 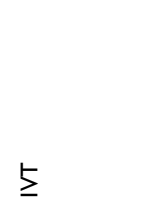 \\
\hline$\Xi$ & ก) & $\tilde{m}$ & $\stackrel{\infty}{\sim}$ & $\hat{\sim}$ & $\stackrel{ \pm}{\sim}$ & 8 & 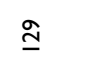 & $\mathcal{F}$ & $\approx$ & $m$ \\
\hline N & $\sim$ & $N$ & N & $N$ & $\sim$ & $\sim$ & N & $N$ & - & $N$ \\
\hline$\underset{\infty}{\infty}$ & 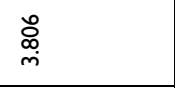 & $\begin{array}{l}\text { त̂̀ } \\
\text { مٌ }\end{array}$ & $\stackrel{\infty}{m}$ & 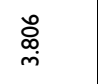 & $\underset{\infty}{\infty}$ & $\underset{\infty}{\sim}$ & ٌْ & $\frac{\hat{n}}{m}$ & $\frac{n}{m}$ & $\hat{m}$ \\
\hline 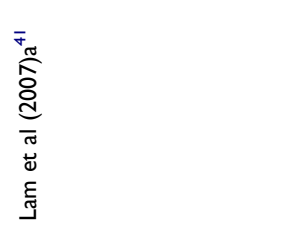 & 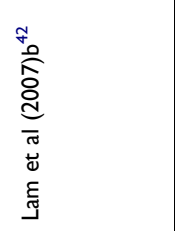 & 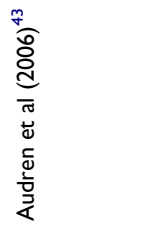 & 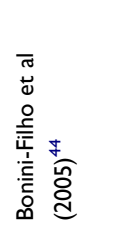 & 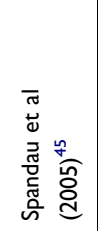 & 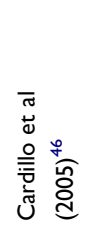 & 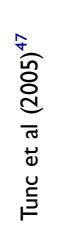 & 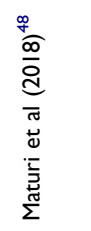 & 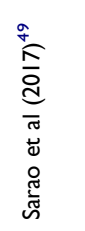 & 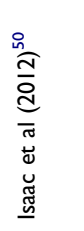 & 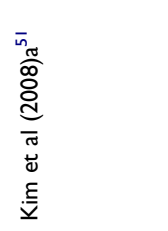 \\
\hline
\end{tabular}




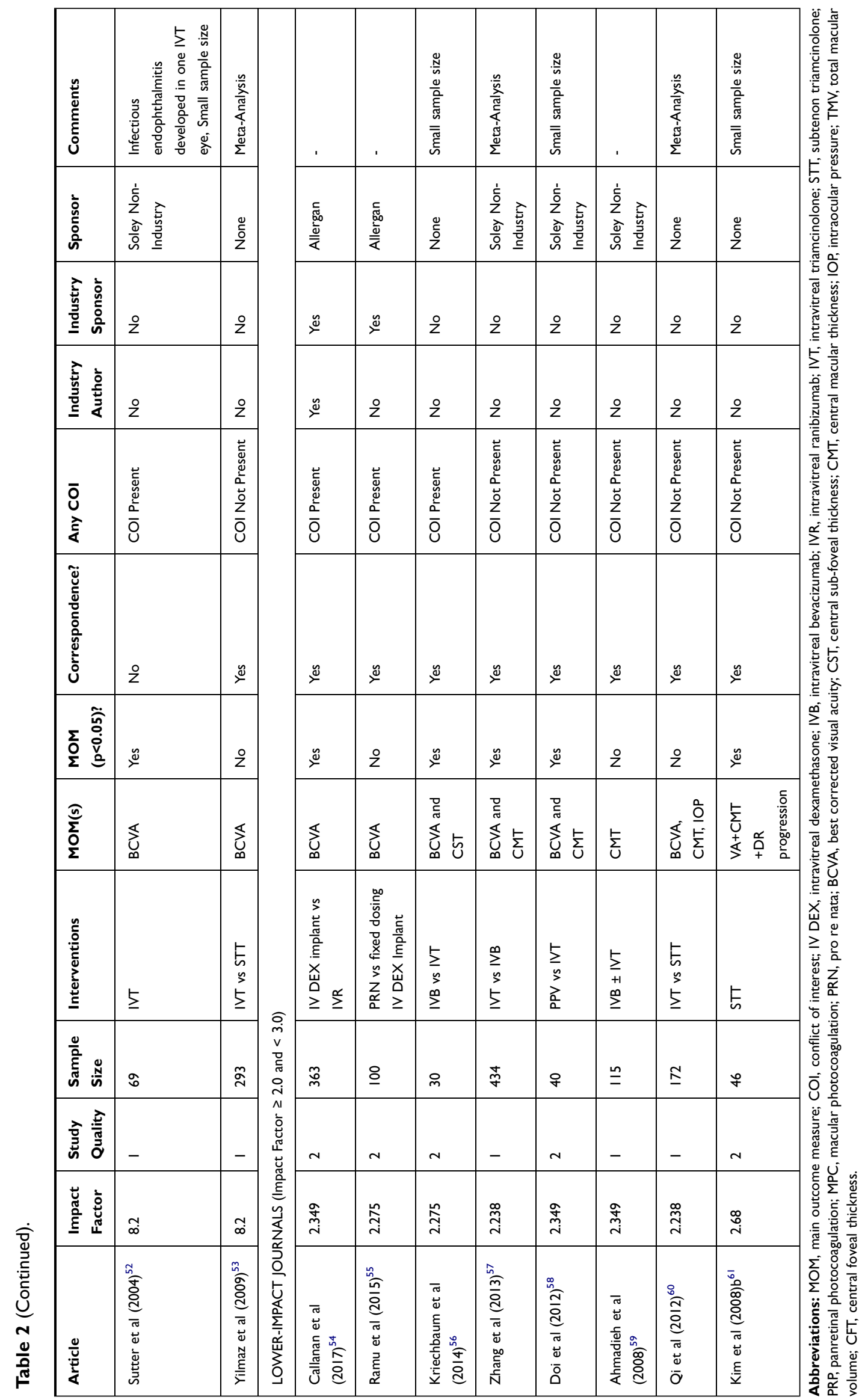


Table 3 Summary of Studies Investigating Steroid Therapy for Diabetic Macular Edema, Based on Funding Status

\begin{tabular}{|c|c|c|c|}
\hline Outcome Studied & $\begin{array}{l}\text { Industry- } \\
\text { Funded } \\
(n=14)\end{array}$ & $\begin{array}{l}\text { Nonindustry- } \\
\text { Funded }(n=30)\end{array}$ & p-value \\
\hline $\begin{array}{l}\text { Correspondence of main } \\
\text { outcome and conclusions }\end{array}$ & $14(100 \%)$ & $27(90 \%)$ & $0.54^{*}$ \\
\hline $\begin{array}{l}\text { Statistically significant } \\
(p<0.05) \text { main outcome }\end{array}$ & $8(57 \%)$ & $18(60 \%)$ & $1.00 *$ \\
\hline $\begin{array}{l}\text { Sample size, mean } \pm \text { SD } \\
(95 \% \mathrm{Cl})\end{array}$ & $\begin{array}{l}374.6 \pm 360.0 \\
(278.4-470.8)\end{array}$ & $\begin{array}{l}82.7 \pm 88.3 \\
(66.6-98.8)\end{array}$ & $0.01^{\dagger}$ \\
\hline $\begin{array}{l}\text { Study quality, mean } \pm \text { SD } \\
(95 \% \mathrm{Cl})\end{array}$ & $\begin{array}{l}1.79 \pm 0.43 \\
(1.68-1.90)\end{array}$ & $\begin{array}{l}1.67 \pm 0.48 \\
(1.58-1.76)\end{array}$ & $0.50^{\ddagger}$ \\
\hline $\begin{array}{l}\text { Journal impact factor, mean } \\
\pm \mathrm{SD}(95 \% \mathrm{Cl})\end{array}$ & $\begin{array}{l}5.90 \pm 2.51 \\
(5.23-6.57)\end{array}$ & $\begin{array}{l}4.68 \pm 2.27 \\
(4.27-5.09)\end{array}$ & $0.14^{\ddagger}$ \\
\hline
\end{tabular}

Notes: *Fisher exact test; '`Student's t-test; ${ }^{\ddagger}$ Mann-Whitney U-test.

Abbreviations: $95 \% \mathrm{Cl}, 95 \%$ confidence interval; SD, standard deviation

Table 4 Summary of Studies Investigating Steroid Therapy for Diabetic Macular Edema, Based on Impact Factor

\begin{tabular}{|c|c|c|c|}
\hline Outcome Studied & $\begin{array}{l}\text { High-Impact } \\
(n=36)\end{array}$ & $\begin{array}{l}\text { Low-Impact } \\
(n=8)\end{array}$ & p-value \\
\hline $\begin{array}{l}\text { Correspondence of main } \\
\text { outcome and conclusions }\end{array}$ & $33(92 \%)$ & $8(100 \%)$ & $1.00 *$ \\
\hline $\begin{array}{l}\text { Statistically significant } \\
(p<0.05) \text { main outcome }\end{array}$ & $21(58 \%)$ & $5(63 \%)$ & $1.00 *$ \\
\hline Industry funding & $12(33 \%)$ & $2(25 \%)$ & $1.00 *$ \\
\hline Any author COI & $14(39 \%)$ & $3(38 \%)$ & $0.76 *$ \\
\hline $\begin{array}{l}\text { Sample size, mean } \pm \text { SD } \\
(95 \% \mathrm{Cl})\end{array}$ & $\begin{array}{l}178.4 \pm 270.3 \\
(133.4-223.4)\end{array}$ & $\begin{array}{l}162.5 \pm 154.1 \\
(108.0-217.0)\end{array}$ & $0.87^{\dagger}$ \\
\hline $\begin{array}{l}\text { Study quality, mean } \pm \text { SD } \\
(95 \% \mathrm{Cl})\end{array}$ & $\begin{array}{l}1.72 \pm 0.45 \\
(1.64-1.80)\end{array}$ & $\begin{array}{l}1.63 \pm 0.52 \\
(1.45-1.81)\end{array}$ & $0.63^{\ddagger}$ \\
\hline $\begin{array}{l}\text { Journal impact factor, mean } \pm \\
\text { SD }(95 \% \mathrm{Cl})\end{array}$ & $\begin{array}{l}5.67 \pm 2.21 \\
(5.30-6.04)\end{array}$ & $\begin{array}{l}2.34 \pm 0.14 \\
(2.29-2.39)\end{array}$ & $<0.01^{\ddagger}$ \\
\hline
\end{tabular}

Notes: *Fisher exact test; ${ }^{\dagger}$ Student's $t$-test; ${ }^{\ddagger}$ Mann-Whitney U-test.

Abbreviations: $95 \% \mathrm{Cl}$, 95\% confidence interval; SD, standard deviation.

and five did not have a disclosure statement. Correspondence between abstract conclusions and significance of main outcome was present in 16 of 17 (94\%) studies with a COI, 21 of $22(96 \%)$ studies without a COI and in four of five $(80 \%)$ studies without a COI disclosure statement $(\mathrm{p}=0.48)$. Studies with a COI had a significantly greater mean sample size when compared to studies without a COI or a disclosure statement $(p<0.01)$. There were no significant differences in rates of significant main outcome measures $(\mathrm{p}=0.25)$, study quality $(\mathrm{p}=0.61)$, or journal impact factor $(\mathrm{p}=0.61)$. These data are summarized in Table 5.

\section{Discussion}

This study aimed to examine whether the presence of industry funding affected the likelihood of biased outcome reporting among studies of intravitreal corticosteroid therapy for DME. Overall, the results of the present study indicated that abstract outcome reporting corresponded with their statistical results for almost all of the included studies, and that funding source was not a predictor for biased reporting. Journal impact factor and the presence of a COI were not predictors of biased outcome reporting. Industry-funded studies and studies with a COI had greater samples sizes but were of similar impact and quality when compared to their counterparts.

The results of this study are reassuring given that biased outcome reporting has been identified in a number of published studies. In 2009, Berwanger et al published the results of a systematic survey of RCT abstract reporting in high-impact general medical journals and found that $29 \%$ of studies lacked a definition of the primary outcome and that half of the studies did not report on side effects or harms. ${ }^{62}$ In RCTs of wound treatments, Lockyer et al found that among studies of wound care treatment that did not have a statistically significant result, $71 \%$ had some form of biased reporting. ${ }^{63}$ Among RCTs in oncology, Vera-Badillo et al found biased reporting of efficacy outcomes to be common in studies with a negative primary endpoint and that toxicity was underreported. ${ }^{64}$ Recently, biased outcome reported has been shown to be prevalent among high-impact neurology journals. ${ }^{65}$

Although biased outcome reporting has been identified as a concern in biomedical research, the role of industry sponsorship has been debated in the literature. Recently, a metaanalysis of "spin" in the medical literature found that clinical trials had the greatest variability in the prevalence of spin, with common practices being detracting from statistically nonsignificant results and inappropriately using causal language. ${ }^{66}$ Although the industry sponsorship was hypothesized by the authors to be associated with spin, the results of this meta-analysis were inconclusive. ${ }^{66}$ Published reviews have found that industry funding was not associated with biased reporting among oncology trials, ${ }^{67}$ musculoskeletal studies, ${ }^{68}$ general medical journals, ${ }^{69}$ or in gastrointestinal research. $^{70}$

Within the ophthalmic literature, Alasbali et al investigated whether funding source was associated with biased abstract conclusions among studies of topical prostaglandins for intraocular pressure lowering. ${ }^{9}$ Their study found $62 \%$ of industry-funded articles to have an abstract conclusion that was not consistent with the results of the main outcome measure, while none of the non-industry-funded articles had noncorrespondence. Additionally, while only $24 \%$ of 
Table 5 Summary of Studies Investigating Steroid Therapy for Diabetic Macular Edema, Based on Any Author COI

\begin{tabular}{|c|c|c|c|c|}
\hline Outcome Studied & Author COI Present $(n=17)$ & No Author COI $(n=22)$ & No COI Disclosure $(n=5)$ & p-value \\
\hline Correspondence of main outcome and conclusions & $16(94 \%)$ & $21(96 \%)$ & $4(80 \%)$ & $0.48^{*}$ \\
\hline Statistically significant $(p<0.05)$ main outcome & II (65\%) & $14(64 \%)$ & I (20\%) & $0.25 *$ \\
\hline Sample size, mean \pm SD $(95 \% \mathrm{Cl})$ & $319.2 \pm 347.7(263.7-374.7)$ & $93.8 \pm 98.8(45.0-142.6)$ & $46.8 \pm 36.3(-55.5-149.1)$ & $<0.01^{\dagger}$ \\
\hline Study quality, mean \pm SD $(95 \% \mathrm{Cl})$ & $1.76 \pm 0.44(1.65-1.87)$ & $1.64 \pm 0.49(1.54-1.74)$ & $1.80 \pm 0.45(1.6-2.0)$ & $0.61^{\ddagger}$ \\
\hline Journal impact factor, mean \pm SD $(95 \% \mathrm{Cl})$ & $5.66 \pm 2.58(5.08-6.24)$ & $4.68 \pm 2.30(4.17-5.19)$ & $4.76 \pm 2.05(3.69-5.83)$ & $0.61^{\ddagger}$ \\
\hline
\end{tabular}

Notes: *Fisher exact test; ${ }^{\dagger}$ One-way ANOVA; ${ }^{\ddagger}$ Kruskal-Wallis test.

Abbreviations: $95 \% \mathrm{Cl}$, $95 \%$ confidence interval; SD, standard deviation.

the industry-funded studies had a statistically significant main outcome measure, $90 \%$ of the industry-funded studies had a proindustry abstract conclusion. ${ }^{9}$ These findings contrast those of the present study quite dramatically and may reflect differences in intervention efficacy (topical prostaglandins vs intravitreal corticosteroids) or differences in methodologies between the studies. Namely, the present study only included RCTs and meta-analyses published in relatively higher-impact journals, which may explain the difference in non-correspondence rates. Recently, our group published a study using a very similar methodology examining the effect of funding source on reporting bias in studies of intravitreal anti-VEGF therapy for retinal vein occlusion. ${ }^{10}$ Similar to the present study, rates of biased abstract reporting were low and were unaffected by funding source, reflecting no differences despite the increased risk profile of intraocular corticosteroids compared to intravitreal anti-VEGF therapy. Finally, the rigorousness of the peer-reviewed process between 2009 and 2019 may partly explain the difference in results. It is interesting to note that all 3 studies with noncorrespondence were published prior to 2008 .

Although the present study found an overall abstract conclusion and study results correspondence rate of $93 \%$, it is important to note that only the primary outcome was evaluated. The most common primary outcomes among the included studies were visual acuity and retinal thickness. Adverse events, namely intraocular pressure elevation, were rarely reported as a primary outcome and are especially relevant in the context of intravitreal steroid therapy. This review identified seven studies that failed to mention the increased prevalence of steroid-related intraocular pressure elevations in their respective abstracts. If this adverse effect was included as reported as a primary outcome in these studies, the overall non-correspondence rate of the present review would have increased by roughly $11 \%$. This highlights the importance of comprehensive outcome reporting to allow readers to fully understand and appreciate the risks and benefits of therapies they later offer to their patients.
The major limitation of the present study was its highly selective inclusion criteria. Unlike prior studies, the present study only included RCTs and meta-analyses that were published in journals with an impact factor greater than 2. RCTs and meta-analyses are considered to provide the highest level of evidence and are likely preferentially assessed by physicians. Although the present study may have excluded highquality studies published in journals of lower impact, the authors feel that this analysis captured articles that would more likely be read by physicians when scanning the recent medical literature. Although the present study did not identify differences in the rates of noncorrespondence between the subgroups of journal impact factor, future studies may find it useful to examine biased reporting among studies published in lower-impact journals (impact factor $<2$ ). Nonetheless, it is reassuring to note that among these higherimpact publications of intravitreal corticosteroid therapy for DME, biased abstract reporting overall appears to be uncommon and unrelated to industry sponsorship or authorship, or to journal impact factor.

\section{Acknowledgment}

The data presented in this manuscript were presented in part at the 2019 Annual Meeting of the American Society of Retina Specialists in Chicago, Illinois.

\section{Funding}

The Bascom Palmer Eye Institute received funding from NIH Core Grant P30EY014801, Department of Defense Grant \#W81XWH-13-1-0048, and a Research to Prevent Blindness Unrestricted Grant. Flaum Eye Institute received funding from Research to Prevent Blindness Unrestricted Grant.

\section{Disclosure}

JS is a consultant for Alcon Laboratories, Alimera Science, Oxurion, Regeneron, and Thrombogenics. AEK is a consultant for Allergan, Alimera Sciences, Regeneron, and Valeant and receives grant funding from Bausch 
Health, Roche/Genentech and Second Sight. The authors report no other conflicts of interest in this work.

\section{References}

1. Lundh A, Lexchin J, Mintzes B, et al. Industry sponsorship and research outcome. Cochrane Database Syst Rev. 2017;2:MR000033.

2. Moses H, Matheson DHM, Cairns-Smith S, et al. The anatomy of medical research: US and international comparisons. JAMA. 2015;313:174-189. doi:10.1001/jama.2014.15939

3. Lundh A, Lexchin J, Mintzes B, et al. Industry sponsorship and research outcome: systematic review with meta-analysis. Intensive Care Med. 2018;44:1603-1612. doi:10.1007/s00134-018-5293-7

4. Bekelman JE, Li Y, Gross CP. Scope and impact of financial conflicts of interest in biomedical research: a systematic review. JAMA. 2003;289:454-465. doi:10.1001/jama.289.4.454

5. Lexchin J, Bero LA, Djulbegovic B, Clark O. Pharmaceutical industry sponsorship and research outcome and quality: systematic review. BMJ. 2003;326:1167-1170. doi:10.1136/bmj.326.7400.1167

6. Schott G, Pachl H, Limbach U, et al. The financing of drug trials by pharmaceutical companies and its consequences: part 2: a qualitative, systematic review of the literature on possible influences on authorship, access to trial data, and trial registration and publication. Dtsch Arztebl Int. 2010;107:295-301. doi:10.3238/arztebl.2010.0295

7. Schott G, Pachl H, Limbach U, et al. The financing of drug trials by pharmaceutical companies and its consequences. Part 1: a qualitative, systematic review of the literature on possible influences on the findings, protocols, and quality of drug trials. Dtsch Arztebl Int. 2010;107:279-285.

8. Sismondo S. Pharmaceutical company funding and its consequences: a qualitative systematic review. Contemp Clin Trials. 2008;29:109-113.

9. Alasbali T, Smith M, Geffen N, et al. Discrepancy between results and abstract conclusions in industry- vs nonindustry-funded studies comparing topical prostaglandins. Am J Ophthalmol. 2009;147:3338.e2. doi:10.1016/j.ajo.2008.07.005

10. Venincasa MJ, Kuriyan AE, Sridhar J. Effect of funding source on reporting bias in studies of intravitreal anti-vascular endothelial growth factor therapy for retinal vein occlusion. Acta Ophthalmol. 2019;97:e296-e302. doi:10.1111/aos.13917

11. Krick TW, Bressler NM. Recent clinically relevant highlights from the diabetic retinopathy clinical research network. Curr Opin Ophthalmol. 2018;29:199-205. doi:10.1097/ICU.0000000000000472

12. Lu L, Jiang Y, Jaganathan R, Hao Y. Current advances in pharmacotherapy and technology for diabetic retinopathy: a systematic review. J Ophthalmol. 2018;2018:1694187.

13. He Y, Ren X-J, Hu B-J, et al. A meta-analysis of the effect of a dexamethasone intravitreal implant versus intravitreal anti-vascular endothelial growth factor treatment for diabetic macular edema. $B M C$ Ophthalmol. 2018;18:121. doi:10.1186/s12886-018-0779-1

14. Whitcup SM, Cidlowski JA, Csaky KG, Ambati J. Pharmacology of corticosteroids for diabetic macular edema. Invest Ophthalmol Vis Sci. 2018;59:1-12. doi:10.1167/iovs.17-22259

15. Fusi-Rubiano W, Blow RR, Lane M, et al. Iluvien ${ }^{\mathrm{TM}}$ (fluocinolone $^{-}$ acetonide $0.19 \mathrm{mg}$ intravitreal implant) in the treatment of diabetic macular edema: a review. Ophthalmol Ther. 2018;7:293-305. doi:10.1007/s40123-018-0145-7

16. Moher D, Liberati A, Tetzlaff J, et al. Preferred reporting items for systematic reviews and meta-analyses: the PRISMA statement. PLoS Med. 2009;6:e1000097. doi:10.1371/journal.pmed.1000097

17. Clarivate Analytics. 2017 Journal Citation Reports; 2017.

18. Heng LZ, Sivaprasad S, Crosby-Nwaobi R, et al. A prospective randomised controlled clinical trial comparing a combination of repeated intravitreal Ozurdex and macular laser therapy versus macular laser only in centre-involving diabetic macular oedema (OZLASE study). Br J Ophthalmol. 2016;100:802-807. doi:10.1136/bjophthalmol-2015-307136
19. Shah SU, Harless A, Bleau L, Maturi RK. Prospective randomized subject-masked study of intravitreal bevacizumab monotherapy versus dexamethasone implant monotherapy in the treatment of persistent diabetic macular edema. Retina. 2016;36:1986-1996. doi:10. 1097/IAE.0000000000001038

20. Maturi RK, Bleau L, Saunders J, et al. A 12-month, single-masked, randomized controlled study of eyes with persistent diabetic macular edema after multiple anti-VEGF injections to assess the efficacy of the dexamethasone-delayed delivery system as an adjunct to bevacizumab compared with continued bevacizumab monotherapy. Retina. 2015;35:1604-1614. doi:10.1097/IAE.0000000000000533

21. Gillies MC, Lim LL, Campain A, et al. A randomized clinical trial of intravitreal bevacizumab versus intravitreal dexamethasone for diabetic macular edema: the BEVORDEX study. Ophthalmology. 2014;121:2473-2481. doi:10.1016/j.ophtha.2014.07.002

22. Callanan DG, Gupta S, Boyer DS, et al. Dexamethasone intravitreal implant in combination with laser photocoagulation for the treatment of diffuse diabetic macular edema. Ophthalmology. 2013;120:1843-1851. doi:10.1016/j.ophtha.2013.02.018

23. Elman MJ, Aiello LP, Beck RW; Diabetic Retinopathy Clinical Research Network, et al. Randomized trial evaluating ranibizumab plus prompt or deferred laser or triamcinolone plus prompt laser for diabetic macular edema. Ophthalmology. 117;2010:1064-1077.e35. doi:10.1016/j.ophtha.2010.02.031

24. Diabetic Retinopathy Clinical Research Network. A randomized trial comparing intravitreal triamcinolone acetonide and focal/grid photocoagulation for diabetic macular edema. Ophthalmology. 2008;115:1447-1449, 1449-10.

25. Diabetic Retinopathy Clinical Research Network. Randomized trial of peribulbar triamcinolone acetonide with and without focal photocoagulation for mild diabetic macular edema: a pilot study. Ophthalmology. 2007;114:1190-1196.

26. Googe J, Brucker AJ, Bressler NM; Diabetic Retinopathy Clinical Research Network, et al. Randomized trial evaluating short-term effects of intravitreal ranibizumab or triamcinolone acetonide on macular edema after focal/grid laser for diabetic macular edema in eyes also receiving panretinal photocoagulation. Retina. 31;2011:1009-1027. doi:10.1097/IAE.0b013e318217d739

27. Campochiaro PA, Brown DM, Pearson A, et al. Sustained delivery fluocinolone acetonide vitreous inserts provide benefit for at least 3 years in patients with diabetic macular edema. Ophthalmology. 2012;119:2125-2132. doi:10.1016/j.ophtha.2012.04.030

28. Pearson PA, Comstock TL, Ip M, et al. Fluocinolone acetonide intravitreal implant for diabetic macular edema: a 3-year multicenter, randomized, controlled clinical trial. Ophthalmology. 2011;118:1580-1587. doi:10.1016/j.ophtha.2011.02.048

29. Elbendary AM, Shahin MM. Intravitreal diclofenac versus intravitreal triamcinolone acetonide in the treatment of diabetic macular edema. Retina. 2011;31:2058-2064. doi:10.1097/IAE.0b013e31822a042a

30. Campochiaro PA, Brown DM, Pearson A, et al. Long-term benefit of sustained-delivery fluocinolone acetonide vitreous inserts for diabetic macular edema. Ophthalmology. 2011;118:626-635.e2. doi:10.1016/ j.ophtha.2010.12.028

31. Gillies MC, McAllister IL, Zhu M, et al. Intravitreal triamcinolone prior to laser treatment of diabetic macular edema: 24-month results of a randomized controlled trial. Ophthalmology. 2011;118:866-872. doi:10.1016/j.ophtha.2010.09.029

32. Takata C, Messias A, Folgosa MS, et al. Intravitreal injection versus subtenon infusion of triamcinolone acetonide during cataract surgery in patients with refractory diabetic macular edema. Retina. 2010;30:562-569. doi:10.1097/IAE.0b013e3181c969b4

33. Mirshahi A, Shenazandi H, Lashay A, et al. Intravitreal triamcinolone as an adjunct to standard laser therapy in coexisting high-risk proliferative diabetic retinopathy and clinically significant macular edema. Retina. 2010;30:254-259. doi:10.1097/IAE.0b013e3181b4f 125 
34. Gillies MC, McAllister IL, Zhu M, et al. Pretreatment with intravitreal triamcinolone before laser for diabetic macular edema: 6-month results of a randomized, placebo-controlled trial. Invest Ophthalmol Vis Sci. 2010;51:2322-2328. doi:10.1167/iovs.09-4400

35. Soheilian M, Ramezani A, Obudi A, et al. Randomized trial of intravitreal bevacizumab alone or combined with triamcinolone versus macular photocoagulation in diabetic macular edema. Ophthalmology. 2009;116:1142-1150. doi:10.1016/j.ophtha.2009.01.011

36. Maia OO, Takahashi BS, Costa RA, et al. Combined laser and intravitreal triamcinolone for proliferative diabetic retinopathy and macular edema: one-year results of a randomized clinical trial. Am J Ophthalmol. 2009;147:291-297.e2. doi:10.1016/j.ajo.2008.08.024

37. Hauser D, Bukelman A, Pokroy R, et al. Intravitreal triamcinolone for diabetic macular edema: comparison of 1,2 , and $4 \mathrm{mg}$. Retina. 2008;28:825-830. doi:10.1097/IAE.0b013e318165767e

38. Ockrim ZK, Sivaprasad S, Falk S, et al. Intravitreal triamcinolone versus laser photocoagulation for persistent diabetic macular oedema. Br J Ophthalmol. 2008;92:795-799. doi:10.1136/bjo.2007.131771

39. Paccola L, Costa RA, Folgosa MS, et al. Intravitreal triamcinolone versus bevacizumab for treatment of refractory diabetic macular oedema (IBEME study). Br J Ophthalmol. 2008;92:76-80. doi:10. 1136/bjo.2007.129122

40. Soheilian M, Ramezani A, Bijanzadeh B, et al. Intravitreal bevacizumab (Avastin) injection alone or combined with triamcinolone versus macular photocoagulation as primary treatment of diabetic macular edema. Retina. 2007;27:1187-1195. doi:10.1097/IAE.0b0 $13 \mathrm{e} 31815 \mathrm{ec} 261$

41. Lam DSC, Chan CKM, Mohamed S, et al. Intravitreal triamcinolone plus sequential grid laser versus triamcinolone or laser alone for treating diabetic macular edema: six-month outcomes. Ophthalmology. 2007;114:2162-2167. doi:10.1016/j.ophtha.2007.02. 006

42. Lam DSC, Chan CKM, Mohamed S, et al. A prospective randomised trial of different doses of intravitreal triamcinolone for diabetic macular oedema. Br J Ophthalmol. 2007;91:199-203. doi:10.1136/ bjo.2006.102848

43. Audren F, Lecleire-Collet A, Erginay A, et al. Intravitreal triamcinolone acetonide for diffuse diabetic macular edema: phase 2 trial comparing $4 \mathrm{mg}$ vs $2 \mathrm{mg}$. Am J Ophthalmol. 2006;142:794-799. doi:10.1016/j.ajo.2006.06.011

44. Bonini-Filho MA, Jorge R, Barbosa JC, et al. Intravitreal injection versus sub-Tenon's infusion of triamcinolone acetonide for refractory diabetic macular edema: a randomized clinical trial. Invest Ophthalmol Vis Sci. 2005;46:3845-3849. doi:10.1167/ iovs.05-0297

45. Spandau UHM, Derse M, Schmitz-Valckenberg P, et al. Dosage dependency of intravitreal triamcinolone acetonide as treatment for diabetic macular oedema. Br J Ophthalmol. 2005;89:999-1003. doi:10.1136/bjo.2004.062596

46. Cardillo JA, Melo LAS, Costa RA, et al. Comparison of intravitreal versus posterior sub-Tenon's capsule injection of triamcinolone acetonide for diffuse diabetic macular edema. Ophthalmology. 2005;112:1557-1563. doi:10.1016/j.ophtha.2005.03.023

47. Tunc M, Onder HI, Kaya M. Posterior sub-Tenon's capsule triamcinolone injection combined with focal laser photocoagulation for diabetic macular edema. Ophthalmology. 2005;112:1086-1091. doi:10.1016/j.ophtha.2004.12.039

48. Maturi RK, Glassman AR, Liu D, et al. Effect of adding dexamethasone to continued ranibizumab treatment in patients with persistent diabetic macular edema: a DRCR network phase 2 randomized clinical trial. JAMA Ophthalmol. 2018;136:29-38. doi:10.1001/ jamaophthalmol.2017.4914

49. Sarao V, Veritti D, Furino C, et al. Dexamethasone implant with fixed or individualized regimen in the treatment of diabetic macular oedema: six-month outcomes of the UDBASA study. Acta Ophthalmol. 2017;95:e255-e260. doi:10.1111/aos.13395
50. Isaac DLC, Abud MB, Frantz KA, et al. Comparing intravitreal triamcinolone acetonide and bevacizumab injections for the treatment of diabetic macular oedema: a randomized double-blind study. Acta Ophthalmol. 2012;90:56-60. doi:10.1111/j.1755-3768.2009.01817.x

51. Kim JE, Pollack JS, Miller DG, et al. ISIS-DME: a prospective, randomized, dose-escalation intravitreal steroid injection study for refractory diabetic macular edema. Retina. 2008;28:735-740. doi:10.1097/IAE.0b013e318163194c

52. Sutter FKP, Simpson JM, Gillies MC. Intravitreal triamcinolone for diabetic macular edema that persists after laser treatment: three-month efficacy and safety results of a prospective, randomized, double-masked, placebo-controlled clinical trial. Ophthalmology. 2004;111:2044-2049. doi:10.1016/j.ophtha.2004.05.025

53. Yilmaz T, Weaver CD, Gallagher MJ, et al. Intravitreal triamcinolone acetonide injection for treatment of refractory diabetic macular edema: a systematic review. Ophthalmology. 2009;116:902-913. doi:10.1016/j.ophtha.2009.02.002

54. Callanan DG, Loewenstein A, Patel SS, et al. A multicenter, 12-month randomized study comparing dexamethasone intravitreal implant with ranibizumab in patients with diabetic macular edema. Graefes Arch Clin Exp Ophthalmol. 2017;255:463-473. doi:10.1007/ s00417-016-3472-1

55. Ramu J, Yang Y, Menon G, et al. A randomized clinical trial comparing fixed vs pro-re-nata dosing of Ozurdex in refractory diabetic macular oedema (OZDRY study). Eye (Lond). 2015;29:1603-1612. doi:10.1038/eye.2015.214

56. Kriechbaum K, Prager S, Mylonas G, et al. Intravitreal bevacizumab (Avastin) versus triamcinolone (Volon A) for treatment of diabetic macular edema: one-year results. Eye (Lond). 2014;28:9-15. doi:10.1038/eye.2013.242

57. Zhang Y, Ma J, Meng N, et al. Comparison of intravitreal triamcinolone acetonide with intravitreal bevacizumab for treatment of diabetic macular edema: a meta-analysis. Curr Eye Res. 2013;38:578-587. doi:10.3109/02713683.2013.767351

58. Doi N, Sakamoto T, Sonoda Y, et al. Comparative study of vitrectomy versus intravitreous triamcinolone for diabetic macular edema on randomized paired-eyes. Graefes Arch Clin Exp Ophthalmol. 2012;250:71-78. doi:10.1007/s00417-011-1777-7

59. Ahmadieh H, Ramezani A, Shoeibi N, et al. Intravitreal bevacizumab with or without triamcinolone for refractory diabetic macular edema; a placebo-controlled, randomized clinical trial. Graefes Arch Clin Exp Ophthalmol. 2008;246:483-489. doi:10.1007/s00417-007-0688-0

60. Qi H-P, Bi S, Wei S-Q, et al. Intravitreal versus subtenon triamcinolone acetonide injection for diabetic macular edema: a systematic review and meta-analysis. Curr Eye Res. 2012;37:1136-1147. doi:10.3109/02713683.2012.705412

61. Kim S-Y, Yang J, Lee Y-C, Park Y-H. Effect of a single intraoperative sub-Tenon injection of triamcinolone acetonide on the progression of diabetic retinopathy and visual outcomes after cataract surgery. $J$ Cataract Refract Surg. 2008;34:823-826. doi:10.1016/j. jcrs.2008.01.018

62. Berwanger O, Ribeiro RA, Finkelsztejn A, et al. The quality of reporting of trial abstracts is suboptimal: survey of major general medical journals. J Clin Epidemiol. 2009;62:387-392. doi:10.1016/j. jclinepi.2008.05.013

63. Lockyer S, Hodgson R, Dumville JC, Cullum N. "Spin" in wound care research: the reporting and interpretation of randomized controlled trials with statistically non-significant primary outcome results or unspecified primary outcomes. Trials. 2013;14:371. doi:10.1186/ 1745-6215-14-371

64. Vera-Badillo FE, Napoleone M, Krzyzanowska MK, et al. Bias in reporting of randomised clinical trials in oncology. Eur $J$ Cancer. 2016;61:29-35. doi:10.1016/j.ejca.2016.03.066

65. Howard B, Scott JT, Blubaugh M, et al. Systematic review: outcome reporting bias is a problem in high impact factor neurology journals. PLoS One. 2017;12:e180986. doi:10.1371/journal.pone.0180986 
66. Chiu K, Grundy Q, Bero L, Boutron I. "Spin" in published biomedical literature: a methodological systematic review. PLoS Biol. 2017;15:e2002173. doi:10.1371/journal.pbio.2002173

67. Peppercorn J, Blood E, Winer E, Partridge A. Association between pharmaceutical involvement and outcomes in breast cancer clinical trials. Cancer. 2007;109:1239-1246. doi:10.1002/cncr.22528

68. Froud R, Bjørkli T, Bright P, et al. The effect of journal impact factor, reporting conflicts, and reporting funding sources, on standardized effect sizes in back pain trials: a systematic review and meta-regression. BMC Musculoskelet Disord. 2015;16:370.
69. Clifford TJ, Barrowman NJ, Moher D. Funding source, trial outcome and reporting quality: are they related? Results of a pilot study. $B M C$ Health Serv Res. 2002;2:18. doi:10.1186/1472-6963-2-18

70. Brown A, Kraft D, Schmitz SM, et al. Association of industry sponsorship to published outcomes in gastrointestinal clinical research. Clin Gastroenterol Hepatol. 2006;4:1445-1451. doi:10. 1016/j.cgh.2006.08.019
Clinical Ophthalmology

\section{Publish your work in this journal}

Clinical Ophthalmology is an international, peer-reviewed journal covering all subspecialties within ophthalmology. Key topics include: Optometry; Visual science; Pharmacology and drug therapy in eye diseases; Basic Sciences; Primary and Secondary eye care; Patient Safety and Quality of Care Improvements. This journal is indexed on PubMed

Submit your manuscript here: https://www.dovepress.com/clinical-ophthalmology-journal
Dovepress

Central and CAS, and is the official journal of The Society of Clinical Ophthalmology (SCO). The manuscript management system is completely online and includes a very quick and fair peer-review system, which is all easy to use. Visit http://www.dovepress.com/ testimonials.php to read real quotes from published authors. 